Poznań

\title{
DAS SANATORIUM DER VERWANDLUNG. ZUR „IMMERWÄHRENDEN“ KREATIVITÄT DER POLNISCHEN REZEPTION VON THOMAS MANNS ZAUBERBERG
}

\begin{abstract}
Sanatorium of Transfiguration. On the "Everlasting" Creativity of the Polish Reception of Thomas Mann's novel 'Magic Mountain'

The reception of Thomas Mann in Poland confirms its special nature, involving a very high degree of transforming processing and extraordinary creativity. It is true that the author of The Magic Mountain, and only he is and was considered in Poland as the German intellectual of the twentieth century par excellence. The more that in the case of this exceptional writer prominent Polish artists and creators of the culture didn't succumb to a superficial and short-lived fascination, but rather they transformed the threads of his works and artistic biography in a highly productive process. Often there was no exaggeration to say about the "reinterpretation" or even a sort of "over-interpretation" of several biographical and literary motifs.

The reception of Thomas Mann was not and is not merely occasional reception at most of an academic character. The rank and importance of eminent Polish writers, literary critics, translators and literary critics presented in the article show in best way the creativity of Polish references and positions taking to Thomas Mann and his work. No other of his novel can compete because of his inspiring role to a permanent creative activity reception area with regard to Magic Mountain. The newest and best prove for it is dating back to 2015 as two adaptation attempts of the novel in the form of opera and theatrical performance were in Poland discussed and extensively commented in the press and on the Internet and finally awarded with major prizes.
\end{abstract}

KEY WORDS: reception, transfiguration, extraordinary creativity, Polish artists and creators, adaptation attempts

Im Jahre 1930 wurde im Zwischenkriegspolen nach einigen Verzögerungen die polnische Übersetzung von Thomas Manns Zauberberg ediert (die polnische Ausgabe war sogar vierbändig). Weil sie ein wachsendes Interesse der polnischen Rezipienten für den Roman nach sich zog, verdankte Thomas Mann den größten Teil seiner überaus starken intellektuellen Resonanz im damaligen Polen nicht unbedingt 
nur seinen bereits von Stanisław Brzozowski intensiv rezipierten Buddenbrooks, sondern vor allem dem endlich in polnischer Sprache zugänglich gemachten Zauberberg sowie den ersten Teilen der Joseph-Tetralogie.

Obwohl sich die Edition der polnischen Übersetzung von Thomas Manns bedeutendstem Roman am Ende der zwanziger Jahre verzögerte und erst drei Jahre nach seinem Warschauer Aufenthalt (12.-15.03.1927) zustande kam, wurde er in der seinen Polen-Besuch flankierenden Zeitungspublizistik - abgesehen von seiner repräsentativen Funktion als Quasi-Botschafter der Weimarer Republik - vor allem als Verfasser des Zauberbergs in der polnischen Literaturpresse überschwänglich gewürdigt:

In dem mehrmals behandelten Zauberberg wird nun die in Elemente zersplitterte Wirklichkeit nach neuen Gesetzen geordnet. Ein Prinzip zu finden, auf Grund dessen dieser Alchimist der Literatur (d. i. Thomas Mann) die überraschenden, manchmal erstaunlichen Verbindungen durchführt, wäre gleichbedeutend mit der Entdeckung der magischen Quellen seines Schaffens. (Reicherówna, 17. April 1927, S. 2) ${ }^{1}$

In ihren ersten Besprechungen und Stellungnahmen haben die polnischen Kritiker auf die singuläre Rolle des Romans im westeuropäischen Kontext hingewiesen und ihn deshalb mit hervorragenden Werken französischer Schriftsteller wie Romain Rolland, Marcel Proust u. a. in eine ehrenvolle Reihe gestellt, was angesichts der damals stark „frankophilen“ Grundorientierung der polnischen Literaturkritik nur als höchstes Lob gedeutet werden konnte. Emil Breiter schrieb dazu:

Thomas Mann artikuliert im literarischen und intellektuellen Bereich der deutschen Ideenwelt die gleichen Werte wie Romain Rolland in der Sphäre des französischen Geistes. (...) So erfüllt die große künstlerische Tat Thomas Manns eine ähnliche Rolle heute, in der Zeit der Begriffsverwirrung, der Verschärfung von Antagonismen, der Spaltung der europäischen Psyche in eine Reihe von abgesonderten und einander feindlichen Ideengehalten. (Breiter, 1927, S. 1)

Thomas Manns Romanwerk wurde als eine „faszinierende und gewaltige Hymne zu Ehren des menschlichen Denkens“ und zugleich als eine ,romantische Vision der dämonischen Hölle“ angesehen, in der „zwei Elemente“, das des Humanitarismus und das des Terrorismus, ,einen erbitterten Kampf um die Seele eines freien Menschen austragen. Die dialektische Kraft dieses Romans sucht ihresgleichen" (ebd.).

Aleksander Guttry sah im Zauberberg einen ,philosophischen und didaktischen Roman", doch als dessen Hauptmotiv betrachtete er die Liebe. Der Zauberberg sei dabei kein Roman für die Massen: „Es genügt nämlich nicht, diesen Roman ein oder zweimal durchzulesen. Im Zuge einer schweren mühseligen Arbeit muss der Geist

\footnotetext{
${ }^{1}$ In diesem, wie auch in anderen Fällen wurden die polnischen Zitate aus der polnischen Thomas Mann-Anthologie Tomasz Mann w krytyce i literaturze polskiej, Poznań 2003 von deren Autor R.D. übersetzt.
} 
ihn verdauen und in jeden seiner Teile eindringen, bevor er ihn ergründen und mit seinem Gefühl durchdringen kann" (Guttry, 1927, S. 218).

Das bedeutendste Zeugnis eines überaus inspirierenden und geradezu zur Kreativität herausfordernden Einflusses des Zauberbergs auf die führenden polnischen Intellektuellen war die bereits nach dem Erscheinen der polnischen Übersetzung verfasste Rezension von Jan Lechoń unter dem Titel Księga prawdy i czaru [Das Buch der Wahrheit und des Zaubers]. Nach Lechońs Meinung wollte Mann auf eine gleichsam Dantesche Art und Weise in einem Werk die ganze zeitgenössische Welt, all ihre Gefühle und Ideen zum Ausdruck bringen, als Projektion ewiger Rätsel vor dem Hintergrund der damaligen Epoche. Zugleich sei Der Zauberberg nicht nur das Buch eines Dichters, sondern auch das eines Wissenschaftlers und Philosophen, wo man neben wissenschaftlichen Neuheiten wie Relativismus und Psychoanalyse auch althergebrachte Begriffe wie Tod und unsterbliche Seele erörtere, die von Thomas Mann stark aufgewertet würden.

Lechoń schrieb auch, viele literarische Motive des Zauberbergs erinnerten ihn an Marcel Proust. Zugleich ließ ihn der Vergleich mit Proust den besonderen Charakter des Mannschen Zeitromans in doppeltem Sinne des Wortes bestimmen. Proust sei ein großer Analytiker gewesen, dagegen konzentriere sich Mann auf eine Synthese. Jede Sache, jede Figur seien nicht nur echt, sondern erhielten eine symbolische Bedeutung - die Liebe von Castorp zu Claudia Chauchat war für ihn ein Meisterstück der psychologischen Analyse, zugleich stand sie symbolisch für das Streben nach Unsterblichkeit. In den späten dreißiger Jahren wurde Manns Romanwerk sporadisch sogar zum Maßstab gewählt, der, an die einheimische polnische Literatur gelegt, dazu diente, ihre Schwächen aufzuzeigen und ihr neue Wege zu weisen (Troczyński, 2003, S. 177-180).

Von der Singularität der polnischen Thomas-Mann-Rezeption, die sich schon in den späten zwanziger Jahren abzuzeichnen begann, zeugt auch die Tatsache, dass die Biographie und das Werk des deutschen Schriftstellers in der polnischen Literatur und Essayistik nicht nur (re)produktiv verarbeitet, sondern sogar, wenn auch vereinzelt, nachgedichtet wurden. In dem 1936 erschienenen Roman Tragiczne pokolenie [Die tragische Generation] von Marian Ruth-Buczkowski spielte die Handlung vier Jahre früher in einem Sanatorium für Lungenkranke. Die Erzählperspektive des Romanhelden war vergleichbar mit der des Romans Jahrgang 1902 von Ernst Glaeser, wobei die zwei Jahre vor Handlungsbeginn herausgegebene Übersetzung des Zauberbergs einen entscheidenden Impuls für den Szenerieaufbau und die Zeichnung einiger Personen des Romans liefern sollte.

Das erzählerische Debüt des damals ganz unbekannten polnischen Autors thematisierte die Tatsache der polnischen Übersetzung des Zauberbergs und lieferte zugleich zusätzliche Argumente für die überaus starke Resonanz des Romans in Polen in den frühen dreißiger Jahren. Als ein anderes erschütterndes Dokument der vielseitigen und kreativen Rezeption des Zauberbergs muss darüber hinaus der pos- 
tum herausgegebene Gedichtband Kotysanka jodtowa [Wiegenlied mit Tannen] (1932) des jung verstorbenen katholischen Dichters Jerzy Liebert betrachtet werden. Nach der Meinung einer engen Bekannten des lungenkranken Liebert, Maria Leszczyńska, hat die Lektüre des Zauberbergs den Dichter zum Verfassen seines tragischen Gedichtzyklus über die Tuberkulose angeregt. Der Zeitpunkt der Diagnostizierung einer schweren Erkrankung bei Liebert fiel dabei genau mit dem Erscheinen der ersten beiden Bände der polnischen Übersetzung des Zauberbergs zusammen. Das von Liebert in Vorahnung des nahenden Todes gelesene Romanwerk sei zu einem derart intimen Erlebnis geworden, dass der Dichter in dem Mannschen Helden Hans Castorp seinen engsten und teuersten Freund zu sehen begann. So habe das als Folge dieser imaginären Freundschaft entstandene, starke Identifikationsgefühl die Entstehung der letzten Gedichte von Liebert entscheidend angeregt.

Die Gruppe der durch die Lektüre des Zauberbergs direkt inspirierten Gedichte umfasste daher Themen, die mit der Krankheit, der menschlichen Existenz und dem Tod verknüpft waren. Den Tod eines Mitpatienten im Sanatorium von Worochta, wo er sich in seinen letzten Lebensmonaten aufhielt, assoziierte Liebert mit dem von Joachim Ziemssen im Zauberberg. Dieser Tod eines an Kehlkopftuberkulose erkrankten Menschen hat ihn indirekt zum Titelgedicht des Zyklus angeregt. In der lapidaren Feststellung: „Im Thermometer wird sich morgen das beschwingte Quecksilber zur Ruhe setzen. An das peinlich sichtbare Vacuum wird sich der Raum bald gewöhnen“ (Jerzy Liebert, Kołysanka jodłowa, Zitat nach Leszczyńska, 1937), spielte Liebert auf die schwerwiegenden Reflexionen Castorps über Raum und Zeit an.

Marceli Ranicki (später Marcel Reich-Ranicki) verglich in seinem in Polen zu stalinistischer Zeit herausgegebenen Buch Z dziejów literatury niemieckiej [Aus der Geschichte der deutschen Literatur] (1953) einige Romane von Thomas Mann miteinander, wobei er zur Konklusion kam, dass die erheblich früher verfassten Buddenbrooks ihm als Literaturkritiker viel näher stünden als Der Zauberberg, weil dieser Roman zwar als das Literaturwerk eines realistischen Schriftstellers, doch auch eines „irrenden Ideologen“ zu betrachten sei (Ranicki, M., 1955, S. 116). Die Buddenbrooks seien aus reinem Realismus heraus entstanden, dagegen mangele es im Zauberberg nicht an angeblichen antirealistischen „Belastungen“, welche Ranicki in erster Linie im Freudismus sah:

Eine übermäßige Beeinflussung durch die Theorie des einst in der bürgerlichen Welt modisch gewordenen Psychologen Sigmund Freud ist wohl im Zauberberg am deutlichsten sichtbar. Einige in diesem Roman enthaltene ausführliche psychologische Analysen, auf falsche Voraussetzungen gestützt und von der Wirklichkeit weit entfernt, behindern hie und da die Aussage dieses Literaturwerks beträchtlich. (Ebd. S. 116-117)

Wenig nützlich war seines Erachtens auch die Überladung des Buchs mit einer großen Zahl von abstrakten Theorien, Konzepten und Erkenntnissen aus verschiedenen Wissenschaftszweigen, die nur die Romanform sprengte und keinesfalls die 
„Ausdruckskraft ihrer hauptsächlichen Aussage“ förderte. Trotzdem verdanke der Leser der Geschichte über Hans Castorp ,eines der reichsten Bilder der bürgerlichen Welt“, weil Der Zauberberg sogar bei allen seinen Fehlern als „ein Abbild der Epoche“ und „beinahe ein Reiseführer durch die Sackgassen der bürgerlichen Ideologie" (sic!) (ebd.) fungiere.

Sehr weit abgesteckt ist in der polnischen Literaturkritik das Referenzfeld für die ideologischen Grundpositionen, welche durch die beiden weltanschaulichen Gegner Settembrini und Naphta repräsentiert werden. Auf diese Hauptopposition berief sich bereits Czesław Miłosz in seinen Romanen Zdobycie wtadzy [Das Gesicht der Zeit] und Rodzinna Europa [West- und Östliches Gelände], wobei er sie viel wichtiger fand als Hans Castorps persönliche Geschichte.

Eine starke Faszination durch den Zauberberg bezeugen die Anfang 2000 edierten Interviewäußerungen des polnischen Science-Fiction-Autors, Philosophen, Essayisten und großen Visionärs der menschlichen Kultur Stanisław Lem (Wikipedia, 2016), der im Rahmen der von Stanisław Bereś geführten Werkstattgespräche eklärte, dass er den Roman Thomas Manns geradezu vergöttere. Seines Erachtens strahlt Der Zauberberg Vigueur und Frische aus, während Doktor Faustus eher nur die Altersweisheit des Autors mit sich gebracht und keine leidenschaftliche Liebe geboten habe. Echt und authentisch ist dagegen nach seiner Meinung die Darstellungsweise der Liebesleidenschaft Hans Castorps zu Frau Chauchat (Lem \& Bereś, 2002, 180-182).

Andererseits hätte man nach der Auffassung Jerzy Jarzębskis - eines ausgewiesenen polnischen Kenners des Gesamtwerks von Stanisław Lem - das Schaffen des polnischen Schriftstellers, darunter vor allem seinen berühmt gewordenen realistischen Roman Szpital przemienienia [Das Krankenhaus der Verklärung] (1948) und dessen enge Verwandtschaft zum Zauberberg nicht verschweigen können. Nach den Worten des polnischen Literaturkritikers verbindet ihn mit dem opus magnum Manns vor allem der ,labormässige“ Charakter des Handlungsorts, welchen das von der Welt abgetrennte Sanatorium darstellt, ferner das erzieherische Motiv mit dem Haupthelden und vor allen Dingen die gelungene Verbindung der zahlreichen Romanabenteuer der Protagonisten mit einer großen Debatte über die philosophischen Themen, die sich gleichzeitig als Versuch einer Diagnostizierung der Lage der europäischen Kultur herausstellt. Der polnische Schriftsteller befasse sich mit dem Zustand des Kulturorganismus, in dessen Rahmen wir zu leben haben, allerdings, ausgehend von einer anderen Position als Thomas Mann, von der Situation eines Menschen nach dem Holocaust, dem in der Weltgeschichte größten Massaker der Menschen, Systeme, Gesellschaften und Ideale aus (Jarzębski, o.J.; Stiller, 2006, S. 16).

Den überzeitlichen und universalen Charakter dieser weltanschaulichen Auseinandersetzung belegte eine Debatte, zu der es nach der Veröffentlichung des Zeitungsbeitrags „Settembrinis veränderte Stimme“ von Stanisław Barańczak im Jahre 1975 kam. Bei diesem Streit ging es ohne Zweifel um das Selbstverständnis des zeitgenössischen Intellektuellen-Künstlers, auch darum, die Legitimitätsmöglichkeiten einer fundamenta- 
listischen Haltung innerhalb eines moralischen Sendungsbewusstseins des Schriftstellers aufzuzeigen. Von der Tragweite des Streits zeugte der Kreis seiner Teilnehmer neben Barańczak beteiligten sich an dieser Polemik Wojciech Roszewski, Krzysztof Karasek und Adam Zagajewski. Ihre Fortsetzung fand sie in den Texten von Leszek Szaruga, Cezary Rowiński und Piotr Bratkowski. Die in den siebziger Jahren entfesselte Debatte bewahrt ihr Aktualisierungspotential bis heute.

Stanisław Barańczak konstatierte in seinem 1975 gedruckten Artikel:

Der Streit zwischen Settembrini und Naphta ist noch nicht erloschen. Diese Diskussion, wohl die wichtigste in der ganzen Literatur des zwanzigsten Jahrhunderts, findet auch heute statt - nicht nur auf den immer aufs neue gelesenen Seiten des Zauberbergs. In Vergessenheit geraten ist die Epoche, in welcher der Streit entstand, einige weitere sind verstrichen, aber in der ohrenbetäubenden Verwirrung lassen sich die Stimmen der beiden Kontrahenten gut erkennen. In anderen Verkleidungen, als andere Personen, mit verschiedenen Kräften ausgestattet, immer noch einander entgegenstehend - der komische, feurige Humanist und der grausam nüchterne Inquisitor. (Barańczak, 2003, S. 264)

Der ,heutige Settembrini”, durch die Poesie vertreten, wich laut Barańczak weit von seinem ursprünglichen Vorbild im Roman ab und hatte mit ihm recht wenig zu tun. Aber er stand immer noch auf der Naphta gegenüberliegenden Seite, und ihr Streit dauerte immer noch an. Und wie Barańczak annahm, könnte ihn, wenn es zu einem endgültigen Duell kommen sollte, auch diesmal vielleicht der scheinbar machtlose Humanist gewinnen, auch wenn sein Schuss - wie im Romantext - nicht einmal den Gegner verletzen würde.

In seiner Polemik warf Wojciech Roszewski Barańczak den Versuch vor, ,verklungene Symbole“ in eine völlig andere historische Dimension zu übertragen: Wer bestimmt bei Barańczak den „korrigierten Settembrini“? Ihn bestimmt restlos der ganz und gar in der ganzen modernen Welt triumphierende Naphta. Ein sanfter Optimismus Barańczaks, der mit dem korrigierten Settembrini im Zusammenhang steht, werde mit einem Fatalismus assoziiert, der mit der ausgeprägten Hypertrophie der Rolle des „korrigierten“ Naphta verquickt sei (Roszewski, 2003, S. 272-273).

Kein Wunder, dass der manchmal so scharfsinnige Kritiker bestimmte Kategorien des Denkens für sich so weit mythologisierte, dass er als Folge einer ziemlich typischen endogenen Angst vor Naphta einen „korrigierten“ Naphta in den Kampf gegen ihn schickt, den Thomas Mann selbst in seinem - gemessen an der historischen Erfahrung - reifsten Roman in Zeitblom umwandelte, der eine zwar notwendige, doch dabei völlig hilflose Figur ist, die ihre Umgebung höchstens durch ihre Schwäche in Erstaunen setzen kann (ebd.). Mögliche Lösungen der Probleme des Schaffens selbst, der moralischen Einstellung des Autors, seiner Verantwortung für die Welt sollte man nach Roszewski woanders suchen: vor allem in der historischen Welt, wo „weder Naphta noch Settembrini Fetische darstellen, wo der Aktivismus der einen den revisionistischen Einstellungen der anderen nicht gegenübergestellt 
wird, wo echte Menschen auftreten, nicht aber nur als Zeichen aus Papier ausgeschnitten" (ebd.).

Damit spielte Roszewski auf den Faust-Mythos an, meinte aber dabei nicht die bittere Version Thomas Manns, sondern diejenige sanftere Form, die ihm Goethe gab. Naphtas Gegner ist Settembrini, aber Mephistopheles' Gegner war Faust. Und doch bedeutete es einen Unterschied in der Qualität.

Ein Jahr später (1976) meldete seine „Zweifel Settembrini betreffend“ Krzysztof Karasek an, der versucht hat, Hans Castorp als dritten Kontrahenten am Streit teilnehmen zu lassen. Ihm zufolge konnte Settembrini von der Sünde des humanistischen Hochmuts nur durch Hans Castorp freigesprochen werden, der als einziger die Menschlichkeit in ihrer vollen Gestalt verkörperte:

Und doch war es eine Sünde, für die Miltons Satan in die Hölle gestürzt und zu Naphta wurde. Ich möchte nicht, dass nach all dem, was hier geschrieben wurde, meine Einstellung mit der Haltung Castorps identifiziert wird. So wie - wahrscheinlich - auch Barańczak sich nicht wünschen würde, dass man seine Haltung mit der Settembrinis identifizierte. Da jedoch bei der Beschreibung des letzteren sich Barańczak die Figur Castorps als Ziel ,setzte“, konnte ich nicht der Versuchung widerstehen, mir auch Settembrini ,zu setzen“. Es wäre jedoch falsch anzunehmen, dass dabei nur meine Bosheit eine Rolle gespielt hätte. Im Ernst, wenn er Settembrini zum Maßstab des modernen Humanismus hätte machen sollen, müsste man praktisch entscheiden, wer Settembrini, wer Naphta sei? Wer sollte darüber entscheiden? (Karasek, 2003, S. 287)

Diese Verfahrensweise schien ihm noch aus einem anderen Grund nicht die richtige zu sein. Sie teilte die Menschen danach ein, wie sie in die Schubladen hineinpassten. Und das wäre nicht abwegig (jede Methode hat ihre dunklen Seiten), wenn nicht die Tatsache störte, dass aus diesen Schubladen zu viele Arme, Beine und Köpfe herausragen: „Man kann zwar die Schubladen zuschließen, wenn Sie es wirklich so gerne wollen, es werden aber diese elenden Gliedmaßen außen vor bleiben, und daran wird nicht einmal die Tatsache etwas ändern, dass diese Schubladen ein Humanist zu schließen sich bemüht“ (ebd.).

Die ganze Debatte versuchte Adam Zagajewski folgendermaßen zusammenzufassen, um sie zu einem vorläufigen Abschluss zu bringen:

Ganz allgemein und oberflächlich formuliert, stört Karasek in Barańczaks Beitrag die eindeutige, rationalistische Einstellung, die Settembrini zugewiesen wird. Die Behauptung von Karasek könnte so lauten: Barańczak nimmt aus der reich innervierten ideellen Symbolik des Zauberbergs nur ein Element heraus, er zeichnet nur eine Gestalt, einen Helden aus, den er, indem er gewisse Merkmale seiner geistigen Ausrüstung ändert, für eine andere Sache und in einem anderen Jahrhundertviertel kämpfen lässt. Settembrini steht für eine humanistische, antitotalitäre Orientierung. Aus einer Romangestalt wird er zu einer Metapher. Barańczak könnte sich sogar einer anderen Metapher bedienen. Nun ja, aber im Allgemeinen verwenden wir Metaphern, mal die eine, mal eine andere. Lass es Settembrini sein. Es ist eine gute Gelegenheit, ein gewisses heikles methodologisches Problem zu umreißen: Können wir zur Artikulierung unserer gegenwärtigen Probleme Elemente aus unserer europäischen Kultur, der alten und neueren, verwenden?"(Zagajewski, 2003, S. 298). 
Keines der Romanwerke Thomas Manns konnte und kann bis jetzt mit der permanenten und schlechterdings zur Kreativität herausfordernden Rezeptionsaktivität hinsichtlich des Zauberbergs verglichen werden. Davon zeugen am besten zwei in das Jahr 2015 zu datierende - ausgiebig kommentierte, diskutierte und schließlich mit hohen Preisen belohnte Versuche, den Zauberberg für die Theaterbühne als Oper und Theaterspektakel zu adaptieren.

Die Theateradaption in der Regie von Wojciech Malajkat erlebte ihre Premiere im Warschauer Theater „Syrena“ am 12. Februar 2015. In den Hauptrollen traten u. a. Patryk Pietrzak (Castorp), Antoni Pawlicki (Joachim Ziemssen), Maria Seweryn (Clawdia Chauchat), Daniel Olbrychski (Mynheer Peeperkorn), Wojciech Malajkat (Lodovico Settembrini), Wojciech Zieliński (Leon Naphta), Przemyslaw Bluszcz (Dr. Behrens), Przemyslaw Glapiński (Dr. Krokowski) und Aleksandra Justa (Carolina Stöhr) auf.

Nach Malajkats geschichtsphilosophischem Adaptionskonzept hätte Der Zauberberg auf der einen Seite die europäische belle époque gekrönt, zugleich aber auch zahlreiche Naturkatastrophen und Plagen vorweggenommen, welche Europa im zwanzigsten Jahrhundert heimsuchten. Malajkat, Regisseur der Vorstellung, schätzte noch vor der eigentlichen Theaterpremiere deren Rolle folgenderweise ein: „Manche sagen, der Erste Weltkrieg sei das Ende der Zauberberg-Zeit und der Beginn der Realität, in welcher das Individuum zu einem wenig bedeutenden Teil einer großen Maschine des Fortschritts, der wirtschaftlichen Entwicklung, des Konsums degradiert wird. Aber auch der Kriegsmaschinerie“ (Syrena.pl, URL). Daher rührte auch sein Protest gegen manche vereinfachende Meinungen der polnischen Theaterkritiker, die wissen wollten, dass die Zeit des Zauberbergs vergangen sei; dass die Gegenwart keinen Ort für die Diskussion über solche Themen wie Leben und Vergänglichkeit darstelle, weil die Moral und die Zeit (die einst nur floss, und jetzt in großer Eile vorbeihuscht) relativ und vergänglich seien (ebd.).

Genau aus diesem Grund kam Malajkat nach vielen Jahren voller Überlegungen und mühseliger Vorbereitungsarbeit auf seinen Lieblingswunsch zurück, auf den Brettern eines Theaters sein Traum-Projekt, die Inszenierung von Thomas Manns Roman, zu realisieren. Auf die Frage, warum er es tue, antwortete er mit den Worten des deutschen Literaturnobelpreisträgers direkt aus dem Roman: „Der wichtigste innere Naturberuf der Menschheit ist ihre Selbstvervollkommnung" (Thomas Mann, Der Zauberberg). Im Zeitraum von hundert Jahren seit seiner Veröffentlichung fand sich kaum ein anderer mutiger Künstler, der es gewagt hätte, sich dieser Aufgabe zu stellen. Derjenige, der schließlich die Herausforderung annahm, gab in den Interviews gern zu, dass die Zauberberg-Inszenierung einer seiner heißesten Träume fast von Kindesbeinen an war. Andere gut unterrichtete Menschen glaubten sogar zu wissen, dass er sich mit dieser Absicht ganze 20 Jahre herumtrug! Er selbst hat in einem Interview mit Izabela Czapska gesagt: „Dieser Roman schmolz in mir im Laufe der Jahre aus“" (ebd.). 
Durch einen Glücksfall erhielt Malajkats Projekt eine Mitfinanzierung aus den Mitteln des polnischen Ministeriums für Kultur und Nationalerbe. Die Schirmherrschaft für die Theaterinszenierung übernahmen übrigens die Botschaft der Bundesrepublik Deutschland in Warschau und der Inhaber der Rechte, der Verlag S. Fischer GmbH, Frankfurt am Main, Deutschland.

Man kann als Kommentar noch hinzufügen, dass auf diese Weise die Geschichte gleichsam einen Kreis schloss (Stopczyk, 2015, URL). Im Jahre 1986 erhielt nämlich ein damaliger Student der Lodzer Filmhochschule die Möglichkeit, die Rolle des Hamlet im renommierten Warschauer „Studio“-Theater zu spielen, dessen Intendant damals Jerzy Grzegorzewski war. Das Spiel leitete ein prominenter Mann des Theaters, der Holländer Guido de Moor, und der als Gastschauspieler auftretende Student hieß Malajkat. Nach 29 Jahren bekam jetzt als Intendant des Warschauer Theaters „Syrena“ nicht nur Wojciech Malajkat selbst, sondern auch ein Absolvent der Lodzer Filmhochschule - Patryk Pietrzak - eine Chance, die Hauptrolle in dem Stück zu spielen, von dem man schon beim Start erwartete, dass es sich in die Geschichte des Theaters fest einschreiben würde, und zwar nicht nur des polnischen! In der Tat stellte die Theateradaption von Tomasz Chyla, Wojciech Malajkat, Marcin Brzozowski in der Regie von Wojciech Malajkat wahrscheinlich überhaupt einen der ersten Versuche dar, diesen für die europäische Kultur und Literatur so fundamentalen Roman auf die theatralische Bühne zu bringen.

Ein weiterer wichtiger Aspekt, der die Entscheidung für die Inszenierungsarbeiten beschleunigte, war Malajkats Faszination für die Person seines ersten Direktors sowie langjährigen Mentors Jerzy Grzegorzewski. Seine kontinuierliche „Verliebtheit" in Bezug auf seinen Theatermeister war in dem im Theater "Syrena“ inszenierten Stück Zauberberg klar zu erkennen. Eben in diesem Theaterstück schien ein gewisser „Grzegorzewski-Geist“" immer noch auf der Bühne „,herumzutaumeln“. Nicht nur im sparsamen Bühnenbild, nicht nur in sehr treffenden Anlehnungen an Grzegorzewski, etwa in den Szenen mit dem vom Publikum abgewandten, im linken Fenster hell beleuchteten nackten Schauspieler, den sich auf der Rückseite der Bühne bewegenden Figuren, vielen Liegestühlen, von denen zwar nur wenige verwendet wurden, die aber alle da waren und die ganze Zeit „mitspielten“, oder in einem Lieblingstrick Grzegorzewskis, welcher auf der Installierung einer Vorbühne auf Kosten der Sitze im Zuschauerraum beruhte.

Weil das Syrena-Theater sein siebzigjähriges Jubiläum beging, beschloss Wojciech Malajkat - Schauspieler, Regisseur und Intendant des Theaters in einer Person -, bei dieser Gelegenheit auf der Bühne einen nicht unbedingt theaterfähigen Text zu reaktivieren. Thomas Manns Zauberberg ist ein Meisterwerk der Literatur, eine universelle Geschichte, die allerwichtigste existenzielle Themen analysiert. Es ist aber auch ein sehr umfassendes Stück Literatur, und für seine verantwortungsvolle Bühnenadaption mussten die erforderlichen Kürzungen vorgenommen werden. Verglichen mit den raffinierten Bühnenbildern und Kostümen, ohne die die Insze- 
nierung ihre ästhetische Form verlöre, erfüllt die Musik von Grzegorz Turnau (es dominieren Werke für Klavier und Violine) nur eine illustrierende Funktion. Sie tritt nur auf, wenn die Figuren ihre tiefsten Emotionen erleben oder gezwungen sind, grundlegende Entscheidungen zu treffen.

Dies waren Details, die mit bloßem Auge zu sehen waren, aber die Hauptsache bestand auch darin, die Schauspieler auf der Bühne zu führen, die einzelnen Szenen und Übergänge unter ihnen festzulegen, die Bühnenbewegung der Schauspieler und ihre Redeweisen zu meistern. Das ganze Stück war als eine Art direkte Huldigung und Danksagung Malajkats an seinen Meister und Mentor, Jerzy Grzegorzewski, gedacht und arrangiert.

Die Schöpfer dieser Inszenierung standen also vor einer der schwierigsten und anspruchsvollsten Aufgaben, nämlich einen Roman in die Sprache des Theaters zu „übersetzen“, der nach bestehenden Ansichten und Ideen als absolut unübersetzbar gilt. Anscheinend hatte der Regisseur Malajkat eine schier bewundernswerte Überzeugungskraft, weil er es geschafft hat, zu dieser mission impossible einige Menschen einzuladen, die solche Herausforderungen mögen.

So wurde das Bühnenbild von Joanna Kuś entworfen; die Musik komponierte Grzegorz Turnau; aber im Großen und Ganzen haben den Erfolg im Wesentlichen zwei Elemente herbeigeführt: Die Textadaptierung wurde von den Autoren des Stücks vorgenommen, und - eine sehr positive Überraschung - für die Kostüme übernahm Tomasz Jacyków die Verantwortung. Das Hauptproblem, welches notwendigerweise gelöst werden musste, um an eine Theaterinszenierung des Romans überhaupt denken zu können, war der Versuch einer Textadaption.

Und es gelang. Wie Tomasz Chyła scherzhaft im Programmheft kommentierte: „Um die Sache ironisch zu pointieren, reduzierte ich die mehr als 1000 Seiten des Originalromans auf 35“. Und das war der Schlüssel, der die Tür öffnete, auch wenn diese Adaption des Zauberbergs schwerlich zu den kürzesten und lapidarsten Theateraufführungen zu rechnen ist.

So dauerte die Inszenierung in Warschau ganze drei Stunden (mit zwei Pausen), doch die Zuschauer haben immer mit einem zusammenhängenden Text zu tun, der eine feste Grundlage für die in kohärenter Reihenfolge ablaufende Handlung bildet. Die orthodoxen Zuschauer konnten zwar bemängeln, dass im Vergleich mit dem Buch einige der Szenen nicht in der richtigen Reihenfolge gespielt, dass einige Passagen von anderen Personen gesprochen wurden, dass der Radioapparat nicht aus der richtigen Epoche stammte, und Clawdia Chauchat so und nicht anders gekleidet war. Dies waren doch absolut keine wichtigsten Details.

Die ganze Inszenierung war sehr gut durchdacht, zügig geleitet und meist sehr gekonnt gespielt. Schon bei der Hauptfigur, Hans Castorp, angefangen, die von Patryk Pietrzak verkörpert wurde. Der junge Schauspieler hat die ihm gegebene Chance, eine so komplexe Rolle in einem so renommierten Stück darzustellen, sehr gut genutzt. Die ganze Zeit spielte er eine ganz charmante junge Person, wobei er 
erst am Ende in einer sehr emotionsgeladenen Szene mit Herrn Albin zeigte, was er wirklich konnte.

Nicht ganz das Gleiche hat man über seinen Cousin, Joachim Ziemssen (Antoni Pawlicki) sagen können. In der literarischen Vorlage war er in der Tat im Militärdienst und träumte die ganze Zeit von einer Rückkehr in den preußischen Armeedienst, doch gleichzeitig betrachtete er den Aufenthalt im Sanatorium als zivilen Ersatz und unterzog sich deshalb mit ganzer Demut und Engagement allen Anforderungen der Medizin. Anders war es auf der Bühne, wo die Zuschauer nur einen Militär vom Typ des mürrischen Feldwebels einer beliebigen Armee sahen, der wütend sein Schicksal und die Umgebung beschimpfte und damit eintönig und vorhersehbar in seinem Verhalten war.

Anders verhielt es sich mit Dr. Behrens (Przemysław Bluszcz), der im Laufe des ganzen Spektakels die Aufmerksamkeit der Zuschauer auf sich zog. Dies war eine weitere Rolle, bei der ein Schauspieler ein großes Plus verzeichnen konnte. Sein Erscheinen auf der Bühne wurde reichlich bejubelt. In ähnlicher Weise wie in der Romanhandlung überwältigte er seinen Assistenten Dr. Krokowski (Przemysław Glapiński), der sehr richtig spielte, aber eigentlich nur auf der Bühne stand. Von den beiden Antagonisten, die um den Geist und die Seele Castorp wetteifern, Lodovico Settembrini (Mariusz Drężek) und Leo Naphta (Wojciech Zielinski), zeigte sich auf jeden Fall Drężek, und zwar nicht nur in einem Pistolenduell, als Sieger. Wie bekannt, war Settembrini Italiener, Schriftsteller, Intellektueller und Gelehrter. All dies wurde auf der Bühne sichtbar, vielleicht mit Ausnahme eines typischen ItalienerBildes, weil Drężek auf die Kraft des Geistes, nicht das südliche Temperament, Wert legte und damit eine sehr gute Rolle lieferte.

In der Rolle der Oberschwester von Myllendonk trat Iwo Pawłowski auf. Zwar erfolgte in dieser Produktion eine Kombination von zwei Figuren des Romans, d. h. der „richtigen“ Oberschwester und einer der Kellnerinnen, aber es war eine tolle Regieidee, und Pawłowski sammelte für sein Spiel reichlich und verdient Applaus.

Von den zwei Frauen, die die Hauptrollen spielten, Clawdia Chauchat (Maria Seweryn) und Caroline Stöhr (Aleksandra Justa), kam letztere dem Original näher. Justa spielte hervorragend die geistig schwankende Frau Stöhr, mit ihren unkontrollierten Lachanfällen. Seweryn, obwohl ganz anders als die Clawdia, fühlte sich aber emphatisch ein in die Rolle einer jungen, dennoch reifen und eleganten Frau der damaligen Zeit, die ein paar Bücher liest, ein wenig verführerisch, sicherlich emanzipiert und fasziniert von der gewalttätigen Männlichkeit ihres Reisebegleiters ist, den sie von ihrer weiten Reise als männlichen Begleiter mit in die Klinik bringt. Dieser „richtige Mann“ ist Mynheer Peeperkorn, verkörpert von Daniel Olbrychski. Weil dieser nicht mehr oft im Alltag auf den Brettern eines Theaters zu sehen ist, war er wie kein anderer geeignet, die Rolle des „diabolischen“ Holländers zu spielen.

$\mathrm{Zu}$ dieser Rollenbesetzung ist zu sagen, dass Olbrychski, indem er Peeperkorn spielte, sein Alter und seine große Kunst- und Lebenserfahrung nutzte, denn ähnlich 
seiner Bühnenfigur hat er diesbezüglich einen enormen Vorsprung vor seiner Umgebung. Im Falle von Daniel Olbrychski und Maria Seweryn kam ein zusätzlicher Beigeschmack zum Vorschein. Vor mehr als zwanzig Jahren (1993) spielten sie nämlich zusammen im Film Kolejność uczuć [Die Reihenfolge der Gefühle] in der Regie von Radoslaw Piwowarski in einer ähnlichen Konstellation als älterer männlicher Meister und junges Mädchen (Julia). Nun, der gesamte dritte Akt wurde dann tatsächlich wie eine One-Man-Show von Olbrychski mit einer bedeutenden Rolle für Seweryn aufgenommen.

Besondere Worte des Lobes verdiente nach übereinstimmenden Meinungen der Theaterkritiker Tomasz Jacyków (Kostüme). Viele Zuschauer erwarteten von ihm jede mögliche Bizarrerie und Schnickschnack. Nichts davon geschah, weil er eine absolut klassische szenografische Umrahmung für die Inszenierung schuf. Sein Bühnenbild war nicht nur in der Zeit der Handlung fest verwurzelt, sondern auch sehr gut auf konkrete Schauspieler abgestimmt. Alle Charaktere trugen feine, hochwertige Kostüme. Die einzige Ausnahme von der literarischen Vorlage bildete nur eine Figur, und zwar die bereits erwähnte Clawdia Chauchat. Ihre Farben waren sehr schön und harmonierten mit dem „sparsamen und kalten“ Bühnenbild.

Daran war auch zu sehen, dass Jacyków über alles im Detail nachgedacht hatte. Was mit der Tatsache zusammenhängen kann, dass auch für ihn Der Zauberberg eins der Lieblings-Bücher ist, das er in einem sehr frühen Lebensalter, eigentlich schon in der Kindheit las. Und diese Faszination durch den Roman war auch auf der Bühne zu sehen. Nur ein Treffen dieser beiden Romanliebhaber, des Direktors (Malajkat) und des Schöpfers der Kostüme (Jacyków), war imstande, so viel positive Energie unter Koautoren und Schauspielern auszulösen, dass dies dann zu einem so überraschend gutem Endeffekt führte.

Nicht zuletzt war die musikalische Umrahmung des Stücks höchst bemerkenswert. Eine besondere Bedeutung hatte das den letzten Teil eröffnende musikalische Motiv aus Richard Wagners Walküre. Dieser „Fremdkörper“ wurde durch Mynheer Peeperkorn eingeleitet. Er antizipierte die nahenden unvermeidlichen Änderungen im Sanatorium, aber auch die kommenden Schrecken des Ersten Weltkriegs.

Der Zauberberg in der Regie von Wojciech Malajkat griff dichotome Themen auf: Leben und Tod, Gesundheit und Krankheit, Leben und Körperlichkeit, das Leben oben und unten. Der letztere Dualismus bezog sich auf zwei Lokalitäten, die im Roman erwähnt werden: auf das echte Leben „da unten im Tiefland“ sowie auf die andere Welt des „Berghof"-Sanatoriums. Die polternden Möbel ließen die Zuschauer bis zur letzten Sitzreihe im Auditorium aufschrecken.

Die Stärke des Textes kam erst im Sprechen von Wojciech Zielinski, Mariusz Drężek, Przemyslaw Bluszcz und Aleksandra Justa (als Caroline Stöhr) zur Geltung. Die von Mann aufgegriffenen universellen Themen - eine allumfassende Erfahrung des Todes, die Zersetzung des Körpers, des Leidens, aber auch des erotischen Triebs, der Liebe und der verblassenden Hoffnung - wirkten nicht mit der ihnen 
zugeschriebenen Kraft. Die Dialoge vermittelten nach Einschätzung der Kritiker keine großen oder extremen Emotionen, sondern wirkten mit ihrer trockenen Rezitation niederdrückend.

So war es auch in den ersten Inszenierungen der Oper, als die Schauspieler noch vor der Aufgabe standen, sich mit dem Text Thomas Manns besser vertraut zu machen, damit die von ihnen gespielten Gestalten den Epochengeist völlig spüren und eine bessere Einsicht in die Emotionen haben, die an ihnen zerren (Wakar, 20. Februar 2015, URL).

Wie Jacek Wakar in seiner Zeitungsrezension feststellte (ebd.), führte der künstlerische Weg Wojciech Malajkats über keinerlei eindeutige Wege. Auf dem Höhepunkt seiner künstlerischen Vollendung entschied er sich, dem Nationaltheater in Warschau den Rücken zu kehren. Er spielte damals Tartuffe in einer herrlichen Aufführung von Jacques Lasalle, hätte auf der nationalen Bühne als einer ihrer wichtigsten Schauspieler bleiben können, aber es sich anders überlegt, weil er nach anderen Chancen und Möglichkeiten suchen wollte. Es war nicht lange nach dem Tod von Jerzy Grzegorzewski, Malajkat wusste, dass ab jetzt alles für ihn anders sein wird. Anstatt an Prestige und Geborgenheit zu denken, ging er sozusagen ins Blaue.

Bei einer anderen Zauberberg-Inszenierung, diesmal in Form einer Oper durch Paweł Mykietyn nach einem Libretto von Małgorzata Sikorski, wurde er zur $\mathrm{Zu}-$ sammenarbeit eingeladen, aber es bedeutete etwas anderes, von außen hinzuzukommen, statt ,fest verankert“ zu sein.

Bislang hatte er in Aufführungen gespielt, etwas Regie geführt, aber es waren nur lauter „Seitensprünge“. Jetzt sollte er für die Bühne in der Litewska-Straße volle Verantwortung übernehmen. Es war das Jahr 2009, und ein Theaterkritiker besuchte Malajkat am neuen Arbeitsplatz. Er entdeckte dort einen kleinen Schrank an der Wand, eine Pinnwand, daran die beiden Schwarz-Weiß-Fotos von Jerzy Grzegorzewski und Marek Walczewski. Der Leiter des Syrena-Theaters hat sie angestarrt und sagte, dass er sie gern als Schirmherren seines Theaters hätte, damit sie ihm beiständen. Er erinnerte an das Stück Das langsame Nachdunkeln der Bilder im Warschauer Studio-Theater.

Es war eine Aufführung wie ein fünfzigminütiges Blitzlicht, herauskonzipiert aus dem großen Roman von Malcolm Lowry Unter dem Vulkan, Regie führte Grzegorzewski, Walczewski spielte damals den Konsul. Malajkat sah sich das Schauspiel mehrere Male an, manchmal aus dem Zuschauerraum, häufiger hinter den Kulissen. Er erzählte, dass er nie eine solche Übereinstimmung zwischen dem Direktor und seinem Schauspieler erlebte - eine Beziehung ohne Worte, bei der eine Geste ausreicht, ein Augenzwinkern, um Bescheid zu wissen und nicht mehr nachfragen zu müssen. Schon damals von Grzegorzewski fasziniert, träumte er davon, dass etwas ähnliches ihn mit seinem Regisseur verbinde. Er wartete einige Zeit, aber nicht zu lange. Jetzt konnte er die Erfüllung seines großen Lebenstraums erleben. 
Bereits im Programmheft zum Zauberberg erwähnte Malajkat seine zwanzigjährige Zusammenarbeit mit dem Direktor, zuerst im Studio-Theater, dann am Nationaltheater. Zwischen 1986, als er einen Gangster aus der Bande in der Dreigroschenoper von Brecht spielte und in ihr Die Moritat von Mackie Messer darbot, und den siebzehn Aufführungen der letzten Inszenierung von Grzegorzewskis Die zweite Rückkehr des Odysseus von 2005 (in der er den Odysseus gab). Es war keine einfache Beziehung Schauspieler-Regisseur. Eher eine Art Initiation: dem jungen Schauspieler Schritt für Schritt die Welt und das Theater zu zeigen, ihm die eigene Vision der Kunst zu enthüllen, und dann den Ausführenden einer Rolle zum Mitschöpfer der szenischen Wirklichkeit zu machen. Ohne Rüstzeug spielte er bei Grzegorzewski fast ausschließlich große Rollen.

Die Aufführung des Regisseurs Malajkat sollte für den 70. Geburtstag der Warschauer Bühne in der Litewska-Straße eine zusätzliche feierliche Umrahmung bilden. Vorher lief hier im Oktober Die Karriere des Nikodemus Dyzma im Stil einer Vorkriegszeit-Revue. Das Geburtstagskind fiel besser in einem „leichten“ Repertoire aus. Przemyslaw Bluszcz hat als Dyzma besser überzeugt denn als Dr. Behrens. Aber zurück nach Davos, wohin uns die auf der Bühne verstreuten Liegestühle entführen. Malajkat hatte nicht den Mut oder die Idee, ein Schauspiel über ein ausgewähltes Problem aus Manns literarischem Werk zu machen. Er versucht nicht die Treue gegenüber dem Autor zu bewahren und „den Roman als Ganzes“ auf der Bühne zu zeigen. Auf der anderen Seite geht das Beste im Zauberberg verloren im Chaos zwischen den eingestreuten Gags, wirkt vage durch die oberflächliche $\mathrm{Zu}$ sammenfassung der Geschichte.

Der Zauberberg von Thomas Mann ist inzwischen zu einem Emblem der hochwertigen Weltliteratur geworden, und auf den Plakaten des Syrena-Theaters mangelte es nicht an Star-Schauspielern: Maria Seweryn, Daniel Olbrychski, Wojciech Malajkat. Für einige hatte die Inszenierung jedoch zu wenig Erfolg. Witold Mrożek warf in seiner Rezension in der polnischen Tageszeitung Gazeta Wyborcza dem Regisseur mangelnden Publikumserfolg (Mrożek, 19. Februar 2015, URL) vor. Wenn man die meisten Direktoren der Warschauer Theater fragen würde, wie ihr Erfolgsrezept sei, würde seines Erachtens die Antwort wahrscheinlich lauten: große Literatur und große Schauspielernamen auf die Bühne zu bringen. Den Zauberberg von Thomas Mann betrachtete man so als ein Sinnbild der großen Literatur - das Schicksal von Hans Castorp, eines Bürgers, Humanisten und Dichters, also eines Deutschen, wie es sich gehört, der im Lungensanatorium in Davos über das Leben, den Tod und die Liebe am Vorabend des Ersten Weltkriegs mitreflektiert.

Warum blieb das Stück also ohne größeren Erfolg? - fragte Mrożek weiter (ebd.). Polnische Quasi-Adaptatoren (Malajkat, Tomasz Chyła und Marcin Brzozowski) taten, was in ihren Kräften stand, um Thomas Manns Roman in einem komplexeren Kontext zu zeigen. Sie begannen die Vorstellung mit der Montage von Radiosendungen, mit der Ankündigung des bevorstehenden Kriegs und dem Unter- 
gang der alten Welt. In den Vordergrund stellten sie den Dialog zwischen zwei Intellektuellen im Widerstreit um die Seele einer jungen Deutschen - den italienischen Humanisten Settembrini und den von der Idee der Revolution faszinierten Jesuiten und Sprecher des „,neuen Mittelalters“ Naphta.

Im Zauberberg sah man darüber hinaus einen Streit über die Ausgestaltung der Weimarer Republik, die gierigen Ideologien zur Beute fiel. Ein Ideendrama, eine geschichtsphilosophische Parabel, und schließlich ein Bildungsroman. Allerdings hat Mann keinen Roman darüber geschrieben, dass die Menschen auf den Liegestühlen hüsteln, pseudointellektuelle Gespräche führen und ab und zu den Liebeskrämpfen verfallen. Oder dass jemand abreist, jemand kommt oder stirbt, bis der Vorhang fällt. In solchen Fällen würden die Rezensenten schreiben, dass der Regisseur aus dem Roman einen Spickzettel machen möchte.

Nur wem konnte dies nützen, wenn es den Zauberberg, wie es scheint, in der Liste der Schullektüren in den polnischen Schulen nicht mehr gibt? Dazu gehört auch Castorps kaum definierte Sexualität - ein in dem Roman wichtiges und subtil durchgeführtes Motiv, welches bei Malajkat in Slapstick-Nummern überarbeitet wurde. Schließlich musste nach der Auffassung des Rezensenten dem boulevardhaften genius loci Tribut gezollt werden. Offensichtlich reichte der als Oberschwester besetzte Iwo Pawlowski als Charakterdarsteller-Zwerg nicht aus. Obwohl er ein schallendes Gelächter bei den Zuschauern schon mit seiner ununterbrochenen Geschäftigkeit auf der Bühne hervorrief.

Einen besonderen Rang nehmen bei Mann Meditationen über die Zeit ein. Die ersten Wochen an einem neuen Ort verlaufen für den Neuankömmling langsam, sie werden tief erfahren und erlebt wie die Kindheit. Später verstreichen ganze Monate wie der Wind. Ein Beispiel für die Relativität der Zeit ist die berühmte Szene mit den sich unendlich verlängernden sieben Minuten Temperaturmessung. Eine größere Herausforderung entsteht, wenn man sich durch drei Stunden des Schauspiels durcharbeiten will. Vielleicht deshalb schlängelte sich auch während der Pause eine lange Warteschlange nach Kaffee in der Cafeteria wie Mäander des Unterbewusstseins des deutschen Bürgers.

Ein kleines Nickerchen zu machen, wurde den Zuschauern des Syrena-Theaters allerdings nicht gegönnt. Im dritten Akt betrat Daniel Olbrychski die Bühne - als Mynheer Peeperkorn, als Liebesrivale Castorps, zerzaust und leidenschaftlich wie der alte Dionysos. Er wirkte komisch und erschreckend. In der Inszenierung des Zauberbergs war Dr. Behrens (Przemysław Bluszcz) beides, Erlöser und Unterdrücker, der das Recht usurpierte, die geistige und körperliche Stärke seiner Patienten zu testen. Ihre Körper diktieren selbst den Fluchtweg, durch unbändige Libido sowie Selbstmord. Die längste Strecke legt Castorp (Patrick Pietrzak) zurück, der zu seinem Vetter Joachim Ziemssen (Antoni Pawlicki) zu Besuch kommt, in Davos sieben Jahre bleibt und den Tod seines Verwandten miterlebt. Auch hier ergreift ihn eine tiefe Neigung zu Clawdia Chauchat, die ihn schließlich zurückweist. So lieferte die 
Adaption von Thomas Manns Roman selbst ein tolles Material für ein interessantes Schauspiel.

In einer etwas späteren Inszenierung der Oper Der Zauberberg von Paweł Mykietyn nach einem Libretto von Małgorzata Sikorska wurden einige Schauspieler der Schwere und dem Gewicht des Textes von Thomas Mann nicht gerecht. Trotz der Universalität und Aktualität der vom Autor aufgegriffenen Themen klangen die Dialoge nicht kraftvoll und eindringlich genug. Die Figuren sprachen die Worte trocknen, und die Emotionen, die sie erleben, scheinen wenig authentisch zu sein, als ob sie sie kaum angehen würden. Dadurch wurde das dreistündige Spektakel zu einer Zeitreise durch die Zeit, sehr anstrengend und langwierig.

In Mykietyns Inszenierung der Oper Der Zauberberg wurde die Geschichte über das Erwachsenwerden und Sterben buchstäblich verkörpert: Die Charakterisierung machte etwa die Sänger zu falschen jungen Männern - sowie zu „falschen Greisen“. Das Stück wurde im Auftrag des Regisseurs Michał Mierczyński für das Theaterfestival Poznań „Malta“ verfasst, wo es im Juni 2015 seine Premiere hatte. Die Autoren beschlossen dabei, ein Apokryph des weltbekannten Romans zu schreiben. Der 23-jährige Diplom-Ingenieur Hans Castorp kommt in das Berghof-Sanatorium in Davos, aber es erweist sich bald, dass das Zimmer, in das er nach einer verstorbenen Amerikanerin einzog, von ihrem Geist nicht verlassen wurde. Eine anonyme Frau wird also zu einer der Heldinnen, die den Kurort aufsuchen, voll von nicht verstorbenen Toten, im Schwebezustand zwischen Leben und Tod. Die Patienten nehmen üppige Mahlzeiten zu sich, zahlreiche Gespräche nehmen eine knappe und abstrakte Form an, und Manns aufstrebende Romankonstruktion wird durch ein Sieb gegeben und auf ihre Konturen, Hauptfiguren und poetische Kürze reduziert.

Ein solcher Perspektivenwechsel hing mit dem Ziel zusammen, den Zauberberg auf zeitgenössische Art und Weise neu zu schreiben. Jeder der vier Autoren hatte eine andere Idee, so dass die Vorstellung nicht nur das Ergebnis der Zusammenarbeit von hervorragenden Individuen, sondern auch ein Feld einer kreativen Dissonanz ist. Die sparsame Regie Andrzej Chyras kontrastierte mit der vielschichtigen Musik von Paweł Mykietyn. Das flache schwarze Bühnenbild Balkas, das der Künstler als „Maulwurfshügel“ bezeichnete, bildete einen Kontrapunkt zu dem Text von Sikorska-Miszczuk. Zu dieser exzentrischen Mischung konnte noch eine andere apokryphe Fassung des Zauberbergs, der Kurzroman Castorp von Paweł Huelle (dazu Dziergwa, 2016), hinzugefügt werden, der eine Variation über Hans' studentische Episode in Danzig imaginiert.

Der Eklektizismus überschattete nicht den Lyrismus, es tauchten sogar bewegende Arien und Ensembles auf, die im zweiten Akt der ganzen Komposition den Beigeschmack des Geheimnisvollen verliehen. Chyras Stück scheint extrem düster mit melancholischen Gesten, konzentriert auf die Beschreibung der Fäulnisprozesse und Bilder von verwesenden Leichen, die der Ohnmacht verfallen. Liebe und Reifwerden sind wie besessen vom Tode gezeichnet. Die elektronische Musik von My- 
kietyn, Balkas funktionale Gerüste und die langsamen Rhythmen, durch Chyras Regie vorgegeben, kommen in die Nähe einer existenziellen Spielart der ScienceFiction-Literatur. Auch für Mykietin war der Zauberberg eins der wichtigsten Bücher seit seiner frühen Jugend. Im ersten Akt beginnt sich alles zu verlangsamen, wie im Roman. Castorp kommt für drei Wochen auf den Berghof, bleibt aber sieben Jahre. Zeit ist in diesem Akt ein illusorischer Begriff, der sich der Kontrolle völlig entzieht. Im Roman dominiert die erotische Atmosphäre. Man will diesen Augenblick so lange wie möglich verweilen lassen.

Im zweiten Akt ist es ganz anders. Langsam tritt man in die Dunkelheit ein. Alles steuert auf eine Katastrophe zu. Man gibt den Helden keine Möglichkeit zu entkommen. Die Helden werden zum Hinrichtungsort geführt. Interessanterweise wurde das Libretto ins Deutsche übertragen. Aber nicht nur. Allerdings tauchen in der Oper viele Sprachen auf. Es gibt Hinweise auf Barockmusik und einen Pop-Track mit dem Text von Alexander Blok. Im Querschnitt werden Konturen in Form eines Maulwurfshügels oder einer Pyramide gezeigt, mit einem Netzwerk von Korridoren, die z. B. zur versteckten Grabkammer führen. Im zweiten Akt wird dieses Objekt umgestürzt, und damit verändern sich seine Funktionen. Die Vertikalität geht in Horizontalität über. Die vertikalen Korridore werden zu einer Art von vertikalen Gräben, wo die Handlung stattfindet. Die Situation vor hundert Jahren ist nicht viel anders als die heutige.

Die Inszenierung der Oper Der Zauberberg von Paweł Mykietyn nach einem Libretto von Małgorzata Sikorska Miszczuk, von Andrzej Chyra und mit Bühnenbild von Miroslaw Balka fand zum ersten Mal während des Theaterfestivals „Malta“ in Poznań (Juni 2015) statt. Das Stück entstand im Auftrag von Michał Mierczyński. Andrzej Chyra, der Direktor des Theaterfestivals „Malta“, wurde für seine künstlerische Darbietung durch die polnische Ministerin für Kultur und Kunst, Małgorzata Omilanowska, mit der Silbermedaille Gloria Artis ausgezeichnet. Die Inszenierung hatte überwiegend positive Rezensionen.

\section{Bibliographie}

Barańczak, S. (2003) Zmieniony głos Settembriniego. In R. Dziergwa (Hrsg.), Tomasz Mann w krytyce i literaturze polskiej. Antologia tekstów $i$ dokumentów (S. 264-270). Poznań: Wydawnictwo Poznańskie (Original: 1975, Literatura, 29).

Breiter, E. (2003). Na przyjazd Tomasza Manna. In R. Dziergwa (Hrsg.), Tomasz Mann w krytyce i literaturze polskiej (S. 162-164). Poznań: Wydawnictwo Poznańskie (Original: 1927, Wiadomości Literackie, 11, 1).

Dziergwa, R. (2016). Polska recepcja Czarodziejskiej góry Thomasa Manna a Castorp Pawła Huellego. Doniosłość 'prologu gdańskiego' w opinii międzynarodowej krytyki literackiej. In M. Wolting \& S. Wolting (Hrsg.), Zrozumieć obcość. Recepcja literatury niemieckojęzycznej w Polsce po 1989 roku. Kraków: universitas.

Guttry, A. (1927). [ohne Titel]. Tygodnik Ilustrowany, Nr. 11, S. 218. 
Jarzębski, J. (o.J.). Skalpel i mózg. Lem.pl. Abgerufen von http://solaris.lem.pl/ksiazki/beletrystyka/szpi tal-przemienienia/127-poslowie-szpital-przemienienia

Karasek, K. (2003). Wątpliwości dotyczące Settembriniego. In R. Dziergwa (Hrsg.), Tomasz Mann w krytyce i literaturze polskiej. Antologia tekstów i dokumentów (S. 279-287). Poznań: Wydawnictwo Poznańskie (Original: 1976, Twórczość, 3, S. 89-97).

Lechoń, J. (2003). Księga prawdy i czaru. In R. Dziergwa (Hrsg.), Tomasz Mann w krytyce i literaturze polskiej (S. 175-176). Poznań: Wydawnictwo Poznańskie (Original: (1930) Wiadomości Literackie, 347).

Lem, S. \& Bereś, S. (2002). Tako rzecze Lem. Ze Stanisławem Lemem rozmawia Stanisław Bereś (2. erweiterte und verbesserte Aufl.). Kraków: Wydawnictwo Literackie.

Leszczyńska M. (1937). Kołysanka jodłowa i Czarodziejska Góra. Tygodnik Ilustrowany, o. S.

Mrożek, W. (19. Februar 2015). Mann nie napisał powieści o ludziach na leżakach. Wyborcza.pl. Abgerufen von http://warszawa.wyborcza.pl/warszawa/1,34861,17452424, Mann_nie_napisal_powiesci_ o_ludziach_na_lezakach_RECENZJA_.html

Piwowarski, R. (Regisseur) (1993). Kolejność uczuć [Film]. Warszawa: Studio Filmowe TOR.

Ranicki, M. (2003). Z dziejów literatury niemieckiej (Fragmenty). In R. Dziergwa (Hrsg.), Tomasz Mann w krytyce i literaturze polskiej (S. 207-222). Poznań: Wydawnictwo Poznańskie (Original: Ranicki, M. (1955). Z dziejów literatury niemieckiej. Warszawa: Wiedza Powszechna).

Reicherówna R. (17. April 1927). Tomasz Mann o sobie. Wywiad własny ,Wiadomości Literackich’. Wiadomości Literackie, Nr. 16, 2.

Roszewski, W. (2003). Cichy głos Serenusa Zeitbloma. In R. Dziergwa (Hrsg.), Tomasz Mann w krytyce $i$ literaturze polskiej. Antologia tekstów $i$ dokumentów (S. 271-275). Poznań: Wydawnictwo Poznańskie (Original: 1975, Literatura, 29).

Stiller, R. (2006). Lemie! Po co umarleś? Kraków: vis-a-vis/Etiuda.

Stopczyk, K. (2015). Okiem obserwatora: Tomasz Mann Czarodziejska góra [reż.] Wojciech Malajkat premiera Teatr Syrena 12 lutego 2015 r. Kulturalanie.waw.pl. Abgerufen von http://kulturalnie. waw.pl/artykuly/1908/tomasz-mann-czarodziejska-gora-\%5Brez.\%5D-wojciech-malajkat-\%E2\%80\% 93-premiera--teatr-syrena.html

Teatr Syrena (2015). Czarodziejska góra. Teatrsyrena.pl. Abgerufen von http://teatrsyrena.pl/lista-spek takli/czarodziejska-gora,s75.html

Troczyński, K. (2003). Thomas Mann. Uwagi porównawcze o literaturze polskiej i niemieckiej. In R. Dziergwa (Hrsg.), Tomasz Mann w krytyce i literaturze polskiej (S. 177-180). Poznań: Wydawnictwo Poznańskie (Original: (1933), Prom, 126-129).

Wakar, J. (20. Februar 2015 ). „Czarodziejska góra” w Syrenie: Propozycja rozmowy o świecie na miarę oryginału Manna. Gazeta Prawna.pl. Abgerufen von http://kultura.gazetaprawna.pl/artykuly/854612, wojciech-malajkat-wychowanek-teatru-grzegorzewskiego.html

Wikipedia (2016). Stanisław Lem [Stichwort]. Wikipedia.de. Abgerufen von https://de.wikipedia.org/ wiki/Stanis\%C5\%82aw_Lem

Zagajewski, A. (2003). Artysta bez właściwości. In R. Dziergwa (Hrsg.), Tomasz Mann w krytyce i literaturze polskiej. Antologia tekstów i dokumentów (S. 299-307). Poznań: Wydawnictwo Poznańskie (Original: (1976) Tygodnik Powszechny, 51). 